EPJ Web of Conferences 19, 04003 (2012)

DOI: $10.1051 /$ epjconf/20121904003

(C) Owned by the authors, published by EDP Sciences, 2012

\title{
Origins of the thick disk of the galaxy as traced by metal-poor stars selected from RAVE
}

\author{
G.R. Ruchtia \\ Max-Planck-Institut für Astrophysik, Postfach 1317, Karl-Schwarzschild-Str. 1, \\ 85748 Garching, Germany
}

\begin{abstract}
Models of the formation of the thick disk of the Milky Way Galaxy make specific predictions about the chemical abundance properties of the metal-weak (and oldest) stellar population in the thick disk. We have undertaken the study of the elemental abundances and kinematic properties of a sample of 319 candidate metal-poor thick-disk stars selected from the RAVE spectroscopic survey of bright stars. Our aim is to differentiate among the present scenarios of the formation of the thick disk. In this study, we measured the abundances of several alpha-elements and found that the thick-disk [alpha/Fe] ratios are enhanced, implying that enrichment proceeded by purely core-collapse supernovae. Further, the sample probes distances ranging out to about $2 \mathrm{kpc}$ from the Sun, allowing for the investigation of the gradients in the thick disk. I will discuss the results from these investigations and how they compare to the predictions made by present models of the formation of the thick disk.
\end{abstract}

\section{INTRODUCTION}

In current cosmological models, structures grow hierarchically in a cold dark matter-dominated universe. In the context of hierarchical clustering, disk galaxies form as a result of significant merging and accretion of smaller dwarf-like galaxies down to redshifts $z<1.0$. Late mergers, as predicted by the $\Lambda \mathrm{CDM}$ models, would not allow extended disk galaxies to form as well as inhibit the formation of exclusively old thick disks. This is in conflict with the old ages found for the Milky Way thick disk [1]. The formation history of the thick disk of the Milky Way can therefore provide constraints on the origins and formation of the Milky Way Galaxy.

Several models of the formation of the thick disk, including scenarios ranging from migration of stars from the inner disk [2] to heating of the thin disk due to mergers [3], have been suggested which make specific predictions about the chemical abundance properties of the metal-weak (and presumably oldest) stellar population in the thick disk. Analyzing the elemental abundance patterns in metal-poor thick-disk stars is therefore extremely important for understanding the formation of the thick disk, and ultimately the Milky Way itself.

Metal-poor stars are intrinsically rare in the (local) disk. A large sample is therefore needed to screen for metal-poor stars. We utilized the Radial Velocity Experiment (RAVE) survey [4]. RAVE is a large, unbiased survey of high $\mathrm{S} / \mathrm{N}$, moderate resolution $(R \sim 7500)$ spectra (wavelength coverage around the Ca-IR triplet) of stars with $I<12$ covering the Southern Hemisphere. All candidate stars were selected from the internal RAVE catalog to have calibrated metallicities $<-0.7$ dex and kinematics consistent with the highest probability of belonging to the thick disk. We then conducted high-resolution spectroscopic observations for 319 of these candidate metal-poor thick stars.

\footnotetext{
ae-mail: gruchti@mpa-garching.mpg.de
}

This is an Open Access article distributed under the terms of the Creative Commons Attribution-Noncommercial License 3.0, which permits unrestricted use, distribution, and reproduction in any noncommercial medium, provided the original work is properly cited. 
EPJ Web of Conferences

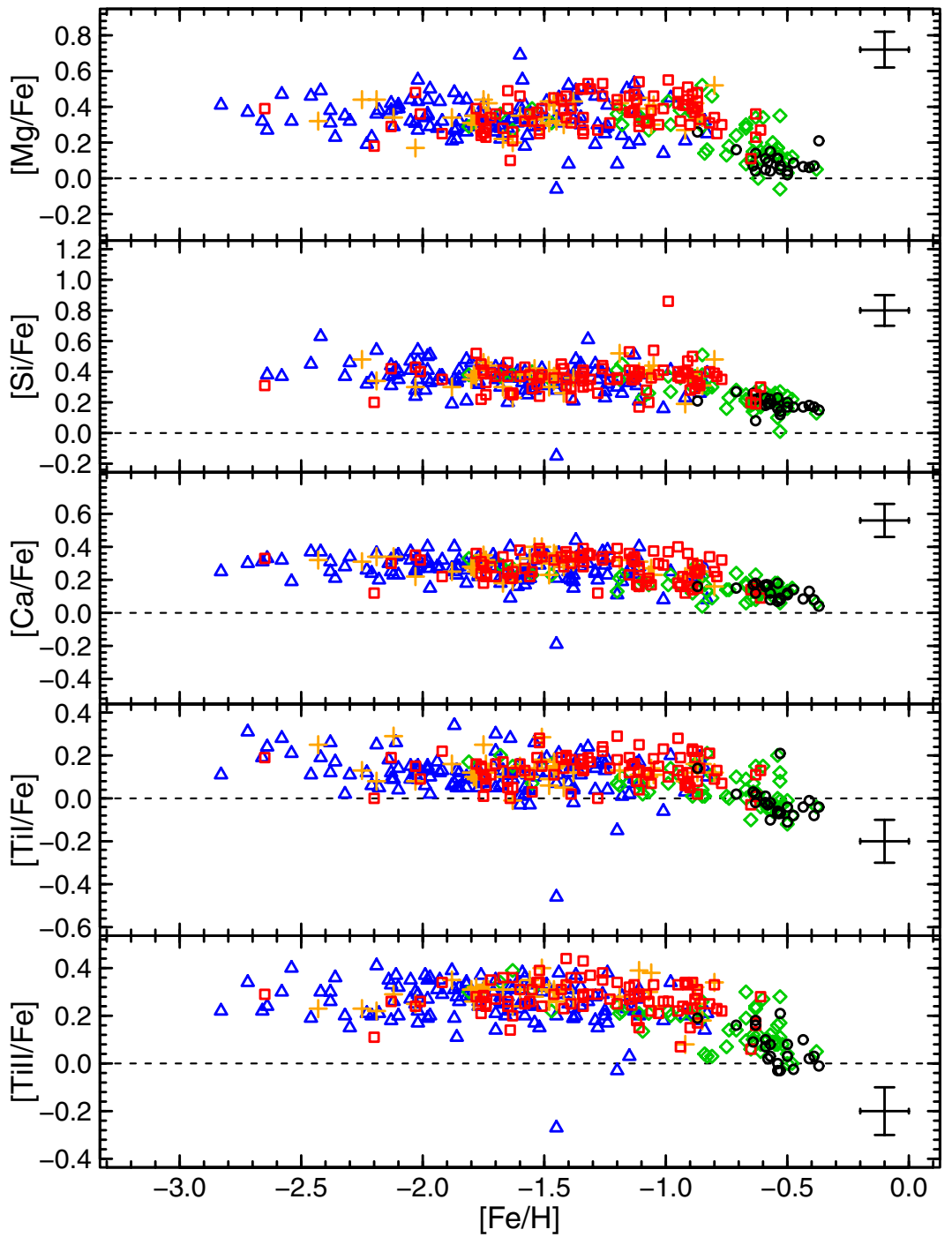

Figure 1. Computed $[\alpha / \mathrm{Fe}]$ ratios vs. $[\mathrm{Fe} / \mathrm{H}]$ for our sample. Note that element ratios are computed using the iron abundance of the same ionization state as the $\alpha$-element (e.g., $[\mathrm{Si} / \mathrm{Fe}]=[\mathrm{Si} \mathrm{I} / \mathrm{Fe} \mathrm{I}]$ ). The black circles, green diamonds, red squares, orange plus signs, and blue triangles correspond to thin disk, thin/thick, thick disk, thick/halo, and halo stars, respectively. The cause of the offset between [Ti I/Fe] and [Ti II/Fe] is most likely nonLTE effects present within the abundance analysis, but the thick disk and halo still show similar enhancement in each.

Full details of our observations, elemental abundance analyses, and Galactic population assignments are given in [5] and [6], while I briefly summarize the findings from these papers below.

\section{PROPERTIES OF THE METAL-POOR THICK DISK}

Our full sample of metal-poor thick disk candidate stars consists of 214 red giant branch (RGB) stars, 31 red clump or horizontal branch stars, and 74 main-sequence or sub-giant stars. Of this sample, 88 were 
assigned to the thick disk, 21 to the thin disk, and 123 to the halo. The remaining stars had probabilities nearly equal between the thin and thick disk or the thick disk and halo.

As shown in Figure 1, those stars assigned to the thick disk have metallicities that range below -2 dex, and have $[\alpha / \mathrm{Fe}]$ ratios that are enhanced and show low scatter $(<0.09$ dex, within our error of 0.1 dex in measured abundances). This indicates that the metal-poor thick disk was pre-enriched by core-collapse supernovae (from an invariant massive-star IMF) during a period of rapid star formation. Further, the similar $\alpha$-enhancement between the thick disk and halo suggests that both were likely pre-enriched by the same massive-star IMF. Additionally, the distribution of orbital eccentricities for our metal-poor thick disk stars (see [6]) does not resemble that of the direct accretion scenario, instead resembling a population that was formed in situ (see [7]). Note, however, that our sample is biased against thick disk stars on high-eccentricity orbits. Further, results presented during talks in this conference suggest that the picture presented in [7] is not so simple.

The $\alpha$-enhancement in the thick disk contrasts with the expectations from models that have direct accretion up until about 6 Gyr ago [8], assuming that the accreted dwarfs formed stars and self-enriched similarly to the surviving dwarfs. All known dwarf spheroidal galaxies typically have extended star formation and lower $\alpha$-enhancement at the same $[\mathrm{Fe} / \mathrm{H}]$ range of the metal-poor thick disk [9]. We can therefore conclude that the late accretion of stars from satellite galaxies did not play a major role in the formation of the thick disk. Direct accretion of stars that formed early ( $\sim 10 \mathrm{Gyr}$ ago $)$ during a period of rapid star formation is, however, still viable.

Due to biases introduced by our candidate selection and analysis technique, we only investigated abundance gradients for those stars assigned to the thick disk with $[\mathrm{Fe} / \mathrm{H}]<-1.2$. The metal-poor thick disk shows a possibly significant vertical metallicity gradient, in which $[\mathrm{Fe} / \mathrm{H}]$ changes by $-0.09 \pm 0.05 \mathrm{dex} \mathrm{kpc}^{-1}$ with vertical height above the Galactic plane. However, a zero-gradient is still possible within our confidence limits. The $\alpha$-enhancement showed no change, both radially and vertically, which further contrasts with models that have a significant amount of direct accretion of stars from satellite galaxies into the thick disk.

\section{CONCLUSIONS}

The metal-poor thick disk of the Milky Way Galaxy is enhanced in the $\alpha$-elements and reaches to metallicities down to -2 dex. We find that the stars in the thick disk most likely formed within the potential well of the Milky Way Galaxy. Direct accretion of stars could have occurred at very early times ( $\sim 1$ Gyr after the start of star formation) in the formation of the thick disk, but the later contribution of accreted stars into the thick disk was very minimal. The abundance trends of the metal-poor thick disk tends to favor models which result in a thick disk with a significant vertical metallicity gradient, however, a uniformly enhanced thick disk is still possible.

I would like to thank the members of the RAVE collaboration, especially Rosemary F. G. Wyse, Jon P. Fulbright, and Gerard Gilmore, without whom this work would not have been possible. Funding for RAVE (www.rave-survey.org) has been provided by institutions of the RAVE participants and by their national funding agencies.

\section{References}

[1] Gilmore, G., \& Wyse, R. F. G., AJ 90, (1985) 2015

[2] Schönrich, R., \& Binney, J., MNRAS 396, (2009) 203

[3] Villalobos, Á., \& Helmi, A., MNRAS 391, (2008) 1806 


\section{EPJ Web of Conferences}

[4] Steinmetz, M. et al., AJ 132, (2006) 1645

[5] Ruchti, G. R., et al., ApJ 721, (2010) L92

[6] Ruchti, G. R., et al., ApJ 737, (2011) 9

[7] Sales, L. V., et al., MNRAS 400, (2009) L61

[8] Abadi, M. G., Navarro, J. F., Steinmetz, M. \& Eke, V. R., ApJ 597, (2003) 21

[9] Tolstoy, E., Hill, V., Tosi, M., ARA\&A 47, (2009) 371 